\title{
A new pen device for injection of recombinant human growth hormone: a convenience, functionality and usability evaluation study
}

This article was published in the following Dove Press journal:

Patient Preference and Adherence

\author{
Maritta Sauer' \\ Carole Abbotts ${ }^{2}$ \\ 'Global Strategic Insights, Merck \\ KGaA, Darmstadt, Germany; \\ ${ }^{2}$ Pharmaceutical Market Research \\ Consultant, London, UK
}

Correspondence: Maritta Sauer Merck KGaA, Frankfurter Str 250, Postcode: FI3I/00I, 64293 Darmstadt, Germany Email maritta.sauer@merckgroup.com
Purpose: Adherence to recombinant human growth hormone ( $\mathrm{r}-\mathrm{hGH})$ is critical to growth and other outcomes in patients with growth disorders, but the requirement for daily injections means that ease of use is an important factor. This study assessed the perceived ease of use and functionality of the prototype of a reusable pen injector (pen device) for $\mathrm{r}$-hGH that incorporates several advanced features.

Participants and methods: Semi-structured 60-minute qualitative interviews were conducted in 5 countries with 57 health care professionals (HCPs) and 30 patients with GH deficiency/ caregivers administering $\mathrm{r}-\mathrm{hGH}$ to patients, including children. HCPs had to be responsible for training in the use of $\mathrm{r}-\mathrm{hGH}$ pen devices and to see $\geq 4 \mathrm{r}$-hGH patients/caregivers per month. Patients/caregivers had to have experience with $\mathrm{r}$-hGH administration for at least 6 months.

Results: Thirty-seven (65\%) of HCPs described the pen device as "simple" or "easy" to use. The aluminum body was generally perceived as attractive, high quality and comfortable to hold and operate. The ease of preparation and use made it suitable for both children and adults. The ability to dial back the r-hGH dose, if entered incorrectly, was mentioned as a major benefit, because other devices need several user steps to reset. Patients/caregivers felt the pen device was easy to use and the injection-feedback features reassured them that the full dose had been given. Overall, 40 (70\%) HCPs and 16 (52\%) patients/caregivers were likely to recommend or request the pen device. Moreover, patients/caregivers rated the pen device higher than their current reusable pens and almost equal to the leading disposable device for ease of learning, preparation, administration and ease of use.

Conclusion: The prototype pen device successfully met its design objectives and was very well accepted by HCPs and patients/caregivers for its ease of use, appearance and functionality.

Keywords: growth hormone deficiency, qualitative interviews, injection feedback, reusable, health care professionals, patients, caregivers, adherence

\section{Introduction}

Recombinant human growth hormone $(\mathrm{r}-\mathrm{hGH})$ is the mainstay of treatment for several growth disorders in children and is also used in the treatment of growth hormone deficiency (GHD) in adults. The treatment regimen requires daily subcutaneous injections over long periods of time, from the age of diagnosis to early adulthood in children with GHD and, for adults with GHD, treatment may be for life. The daily injections and long treatment duration inevitably lead to difficulty in maintaining adherence. ${ }^{1-3}$ For children in particular, maintaining adherence to treatment is of paramount importance, because poor adherence will adversely affect final adult height. Although adherence is difficult to monitor accurately, a number of studies have clearly 
indicated that poor adherence is a particular problem for some patients that can adversely affect clinical outcomes and requires additional input from caregivers. ${ }^{1,3-5}$

The key factors associated with poor adherence to daily injections are the discomfort associated with injections, dislike of needles, the prolonged treatment period and the complexity of administering the correct dosage. ${ }^{1,6-8}$ Individual and family dynamics and the patient or their family's understanding of the benefits of treatment and the consequences of poor adherence or non-adherence may also play a role. ${ }^{1,6}$ Other factors known to impact on adherence to $\mathrm{r}-\mathrm{hGH}$ therapy include the type of delivery system, cost of treatment, socio-economic status, lack of communication from health care professionals (HCPs), the lack of immediate perceptible benefit causing treatment fatigue, and peer or psychosocial pressure. ${ }^{1,7,8}$

A number of different types of injection device have been developed to improve acceptability and ease of use in comparison with the traditional needle and syringe. These include syringes with hidden needles, disposable pre-filled injector pens, reusable injector pens, an electronic auto-injector, and needle-free injectors. ${ }^{6,9}$

A prototype of a new reusable injection pen (pen device) is now at an advanced stage of development for use with r-hGH (Saizen ${ }^{\circledR}$ [Merck KGaA, Darmstadt, Germany]), although this pen is not yet approved or available. The new pen device comprises a multi-dose injection mechanism constructed of aluminum, together with a multi-use cartridge system, a full cartridge-viewing window, a single-digit dosedisplay window with a rotating dose-selection knob and an injection button (Figure 1).

The new pen device was designed to improve on the currently available device on the ease of use and appearance, with optimized dose-display contrast (black text on a white background), to be robust over time and to have increased functionality for administering injections of r-hGH to patients with GHD. These factors have a key role in the acceptability of an injection device to a patient, which, in turn, can have a major influence on their adherence to the demanding injection regimen inherent in $\mathrm{r}-\mathrm{hGH}$ therapy. ${ }^{6}$
The aim of the study reported here was to assess the extent to which potential users of the new pen, patients/ caregivers or HCPs considered the device to meet the design brief. Most of the HCPs were nurses who are likely to be involved in recommending the pen device and/or training patients in its use. A secondary aim of the study was to assess how effective the written instructions were in the Quick Reference Guide provided with the pen device, which will form the basis of the instructions for use for the pen device. This was achieved by observing how easily the participants could prepare the pen device, insert the cartridge, set a prescribed dose and inject into an injection pad, while reading the Quick Reference Guide. No specific training in the use of the new pen device was provided, although participants may have received training previously in the use of pen devices. Finally, the relative importance of the selection criteria used by caregivers, patients and HCPs when choosing a pen device was assessed.

\section{Participants and methods Design and data source}

This multi-country study was conducted in the USA, France, Germany, Brazil and South Korea. The study was initiated in the USA (5 sites) in December 2015 and in France (3 sites), Germany (4 sites), Brazil (2 sites) and South Korea (1 site) in March 2016. The US study was completed in January 2016 and all others in April 2016. Semi-structured 60-minute qualitative interviews were conducted at each site with HCPs and caregivers responsible for administering r-hGH to children with GHD (PGHD) and to adults with GHD. HCPs were selected from nurses, endocrinologists, GH coordinators (GHCs) and medical assistants (MAs).

The study was conducted according to the European Pharmaceutical Market Research Association Code of Conduct, ${ }^{10}$ and all participants provided written informed consent before participation in any aspect of the study.

Criteria for selection of HCPs included senior grade with $\geq 3$ years of experience and personal responsibility either for initiating treatment in patients with GHD or training individuals on how to administer $\mathrm{r}-\mathrm{hGH}$. They must also have

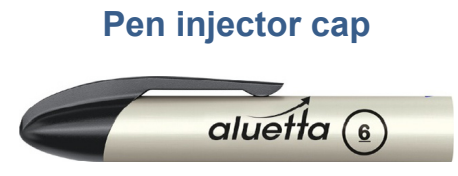

Figure I Components of pen device.

Note: Saizen ${ }^{\circledR}$ is manufactured by Merck KGaA, Darmstadt, Germany.

\section{Pen injector body}

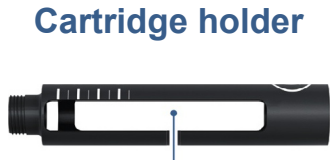

Cartridge window 
administered and/or trained patients or carers/parents to use a r-hGH device for GHD, either in children or adults, within the previous 3 months.

Criteria for selection of caregivers of PGHD patients were having personal responsibility either for injecting or supervising the injection of $\mathrm{r}-\mathrm{hGH}$ with a pen or other device for at least 6 months. In Brazil and South Korea, users with previous experience of syringe and vial use were also eligible for the study.

For the purposes of this study, participants were asked to assume that the pen device was approved by the relevant regulatory authorities and was available for use. The participants were all provided with the pen device (Figure 1), which was red in the USA and silver in the other countries. They were also given a Quick Reference Guide leaflet (Figure 2) that explained the usage and handling of the pen device. Participants were allowed time to comment on the look and feel of the pen device and then, while reading the Quick Reference Guide, were asked to prepare the pen device by inserting a dummy $\mathrm{r}-\mathrm{hGH}$ cartridge, attaching a pen needle and priming it. They were then asked to administer the injection into an injection pad.

\section{Endpoints}

Participants recorded their perceptions of the performance of current devices on a scale ranging from 1 to 7 , in which $1=$ not at all easy and $7=$ very easy. Participant likelihood of recommending the new pen device was evaluated using a $0-10$ Juster numeric scale, in which $0=$ extremely unlikely and $10=$ extremely likely. ${ }^{11}$ The intermediate scores were defined numerically in both scales.

Trained observers recorded comments made by the study participants, observed and recorded how they interacted with the pen device, and assessed and recorded how well they had comprehended and implemented the instructions in the Quick Reference Guide. Demonstration of the use of the pen device was given after this initial assessment, based on the usual training techniques used in each of the study countries.

\section{Results}

A total of 57 HCPs and 30 patients/caregivers participated in the study in the 5 countries involved (Table 1).

In terms of initial perceptions, 15 (26\%) HCPs chose the adjective "sleek" to summarize the appearance of the pen device. "Modern" and "simple" were the words most commonly chosen by caregivers/patients to describe the pen device. The relative frequency of words used by all participants to describe the pen device is represented visually in a word cloud (Figure 3): the larger the word, the more frequently it was used.
The pen device was perceived as comfortable to hold and operate. Twenty-one (70\%) patients/caregivers spontaneously mentioned both its weight and size as favorable features and stated that the aluminum pen device and cap felt sturdy and robust. Fifty-four (62\%) of all respondents spontaneously mentioned the look and feel of the pen device as a favorable attribute. The aluminum body was considered particularly attractive, providing a high-quality finish in comparison with devices made of plastic.

Thirty-seven (65\%) HCPs described the pen device as "simple" or "easy" to learn, teach or use; 22 (39\%) used the words "accurate" or "reliable". The trained observers noted that 27 (90\%) patients/caregivers dialed the dose accurately the first time they handled the pen device. The injection button was considered smooth, firm and easy to press - "so easy a child could use it" - with a reassuring click when the injection was complete. Less pressure was required to carry out the injection compared with other devices, so the whole injection process felt controlled. Moreover, the speed of injection was perceived to be optimal - neither too slow nor too abrupt. The patients/caregivers appreciated seeing the plunger as it moved through the cartridge window.

The accompanying dose countdown to zero during the injection process was highlighted as a benefit by all respondent types, as it satisfied them that the correct dose had been delivered. Fifty-two (60\%) of all respondents spontaneously mentioned the process involved in setting the dose as a favorable feature of the pen device. The ability to dial back the dose if it was initially set incorrectly was seen as a key benefit, and the setting of the dose in increments of $0.1 \mathrm{mg}$ was seen to enable accurate dose selection.

Although inserting or changing a cartridge meant an additional step for previous users of disposable devices, the reusable nature of the pen device was overall seen to reduce waste and contribute to the protection of the environment. It was also considered easy to insert or change the cartridge and view the quantity of $\mathrm{r}-\mathrm{hGH}$ remaining.

Compared with the current market leaders in disposable and reusable devices, globally HCPs rated the pen device highly (Figure 4). Among HCPs in all countries, overall ease of use was seen as the most important factor in device selection (Figure 4). The pen device was considered to perform successfully in terms of being both easy to prepare and easy to teach or learn how to use. In addition, HCP ratings of the pen device's ease of preparation/injection were almost as high as those for the leading disposable device (Figure 4).

Of the negative comments about individual device features reported by all respondents, 38 (44\%) commented on the small size of the dose window compared with other devices 


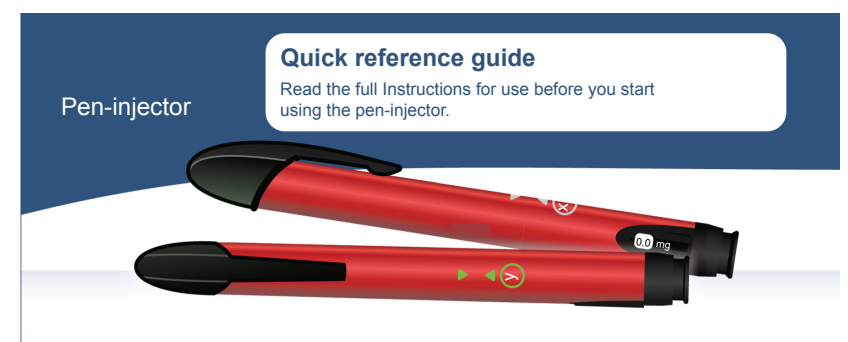

Step 1: Get ready

a Wash hands.

(b) Match pen-injector and cartridge dose types.
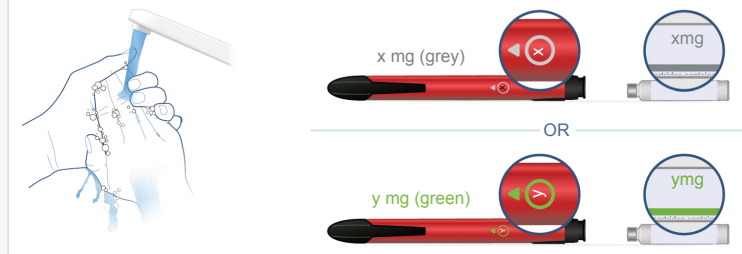

\section{Step 2: Inspect your cartridge}

Inspect cartridge is NOT damaged, and liquid is clear, colorless and does NOT contain particles.
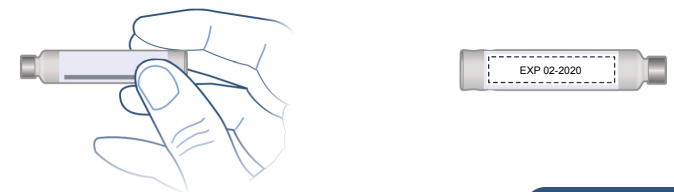

Continue on inside

\section{Step 3: Insert your cartridge}
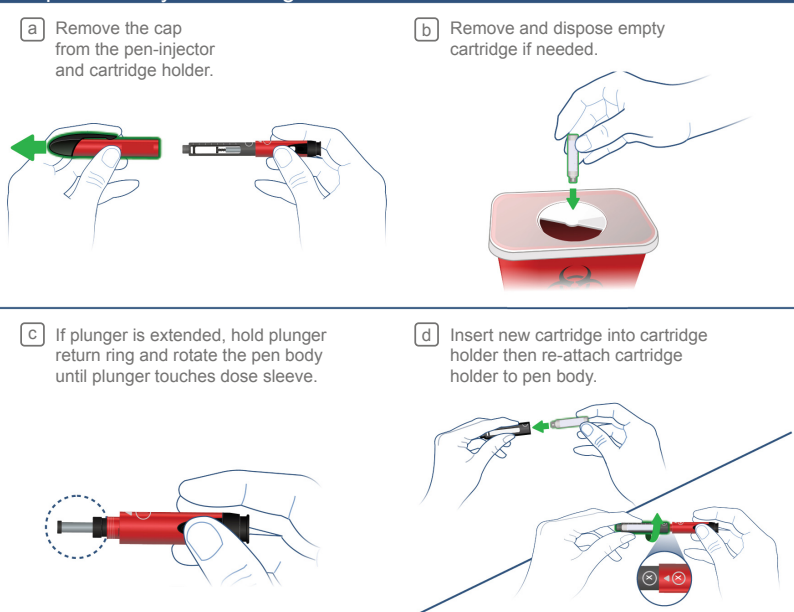
Id Insert new cartridge into cartridge
holder then re-attach cartridge holder to pen body.

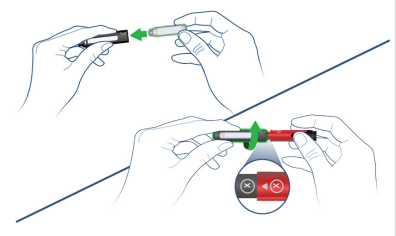

\section{Step 4: Attach your needle}

a. Hold a new needle in one hand and peel off protective seal; reserve the cap for later.
b] Hold outer needle cap straight and screw it onto cartridge holder unt
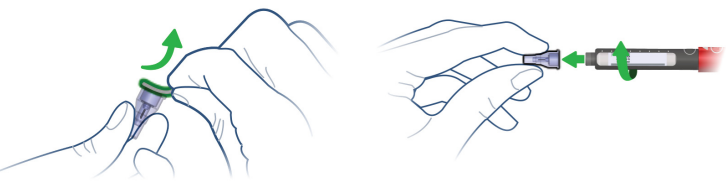

Step 5: Choose and prepare your injection site

a Select an injection site. b. Clean the injection site with an
alcohol pad.
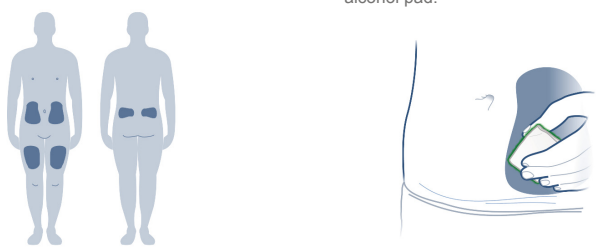

\section{Step 6: Prime your pen-injector}

\section{IMPORTANT: Only prime the pen-injector when starting a new cartridge.}

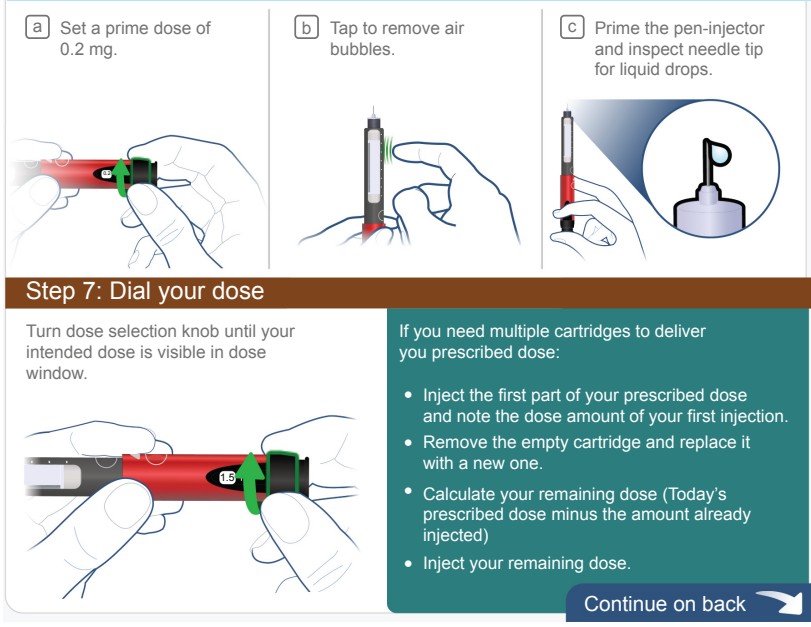

\section{Step 8: Inject your dose}

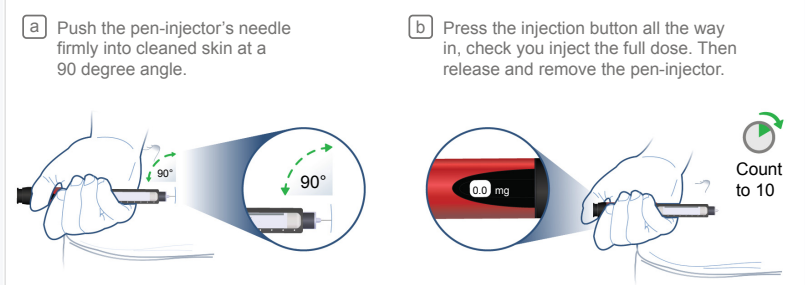

Step 9: Remove and dispose needle
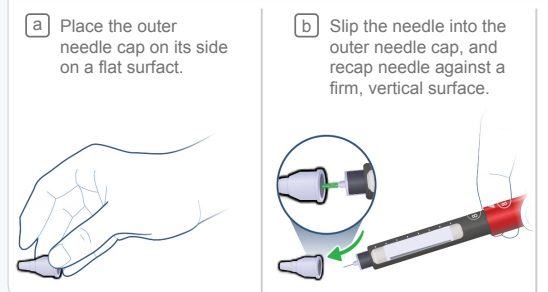
C. Unscrew capped needle,
remove it and dispose it in a sharps container.

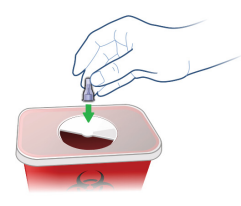

Support and questions

If you have questions or problems using the pen-injector, please contact your healthcare provide

Or call patient support services at:

c \#\#\#-\#\#-\#\#\#\#

Figure 2 Quick reference guide to use of the pen device.

Notes: Saizen ${ }^{\circledR}$ is manufactured by Merck KGaA, Darmstadt, Germany. 
Table I Study participants by type in each country

\begin{tabular}{llllll}
\hline Participants & USA & France & Germany & Brazil & $\begin{array}{l}\text { South } \\
\text { Korea }\end{array}$ \\
\hline HCPs $(n=57)$ & 22 & 7 & 8 & 12 & 8 \\
$\begin{array}{l}\text { Care givers, AGHD } \\
\text { patients }(n=30)\end{array}$ & 5 & 7 & 6 & 6 & 6 \\
$\begin{array}{l}\text { Total respondents } \\
\text { Mtal }\end{array}$ & 27 & 14 & 14 & 18 & 14 \\
\hline
\end{tabular}

Abbreviations: AGHD, adults with growth hormone deficiency; HCPs, health care professionals.

that show 3 numbers in the window, and 23 (26\%) found the dose-setting knob was slightly stiff to turn. These comments related to features specific to the prototype device that will be rectified in the final version of the device. When HCPs were questioned about selected characteristics of the pen device, 29 (51\%) had negative comments on aspects of the plunger rod (instructions were not clear, the rod looked delicate and it needed to be rewound); however, for other device features, the favorable comments far outweighed any reservations (Figure 5), and any minor concerns were not perceived as potential barriers to full adoption of the pen device.

Patients/caregivers rated the pen device higher than their current devices in terms of ease of learning, preparation, administration and ease of handling/use (Table 2). The pen device was perceived as easier to use than the leading reusable device by both HCPs and caregivers familiar with this device. The pen device was seen as easy to prepare and use, making it suitable for both children and adults to

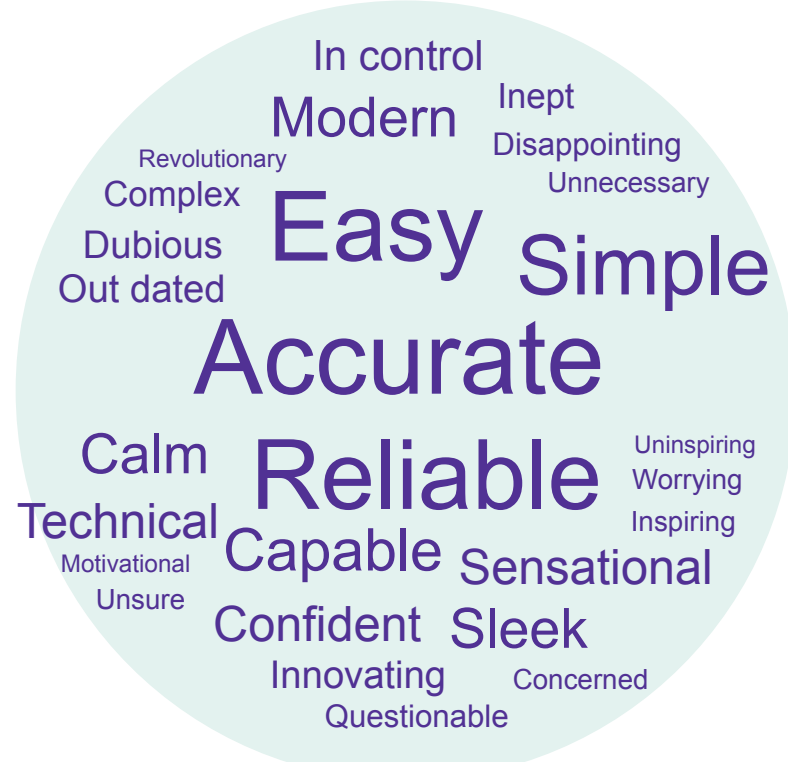

Figure 3 Word cloud showing relative frequency of words used to describe the pen device by $\mathrm{HCPs}$ and caregivers combined.

Abbreviation: $\mathrm{HCPs}$, health care professionals. self-administer (Table 3). The sleek, distinctive appearance of the pen device, combined with its ease of preparation/injection, was felt to enhance the overall user experience and to differentiate the pen device from other reusable pens (Table 3).

Forty $(70 \%)$ HCPs stated that they were likely to recommend (categories 8-10) the pen device to patients (Figure 6A), with the likelihood highest in Brazil and the USA. Similarly, 16 (52\%) patients/caregivers stated that they would request the pen device from their HCP (categories 8-10) when it became available, to replace the device they currently use (Figure 6B).

\section{Discussion}

Good adherence is crucial to obtaining optimal growth outcomes with r-hGH therapy in children and is also important in normalizing body composition, aerobic exercise capacity and quality of life, lipid and carbohydrate metabolism and cardiovascular function, both in children and adults with GHD. ${ }^{5,8,12}$ The acceptability and usability of the injection device is clearly of paramount importance in obtaining and sustaining good adherence to a demanding daily or 6-times-aweek injection regimen. This study was conducted to assess the acceptability, functionality and ease of use of a prototype of a novel pen device to HCPs, caregivers or patients, prior to it being made available.

Prior to testing, HCPs and caregivers/patients were not given any training specific to this pen device, although they may have previously received training in the use of other pen devices. The reason for this was that a secondary aim of the study was to evaluate the usability of the Quick Reference Guide provided with the pen device. However, because the normal practice for training patients to use r-hGH injection devices varies across different countries and regions, according to the health system involved, some patients may have received more training than others. In the USA, device training will be given by nurses or doctors. Nurses are generally able to spend more time than doctors with their patients on the training and monitoring of device usage and can develop good relationships that can help maintain adherence to treatment. Time spent in training ranges from 5 to 60 minutes for the initial session, although this could take as long as 2 hours if the patient is new to injecting or asked a lot of questions. The time taken also depended on whether the training was for adult patients or caregivers or whether a child old enough to start self-injecting under supervision was being taught. Prior to the training session, the pen device and/or starter kit was shipped to the patient or caregiver's 


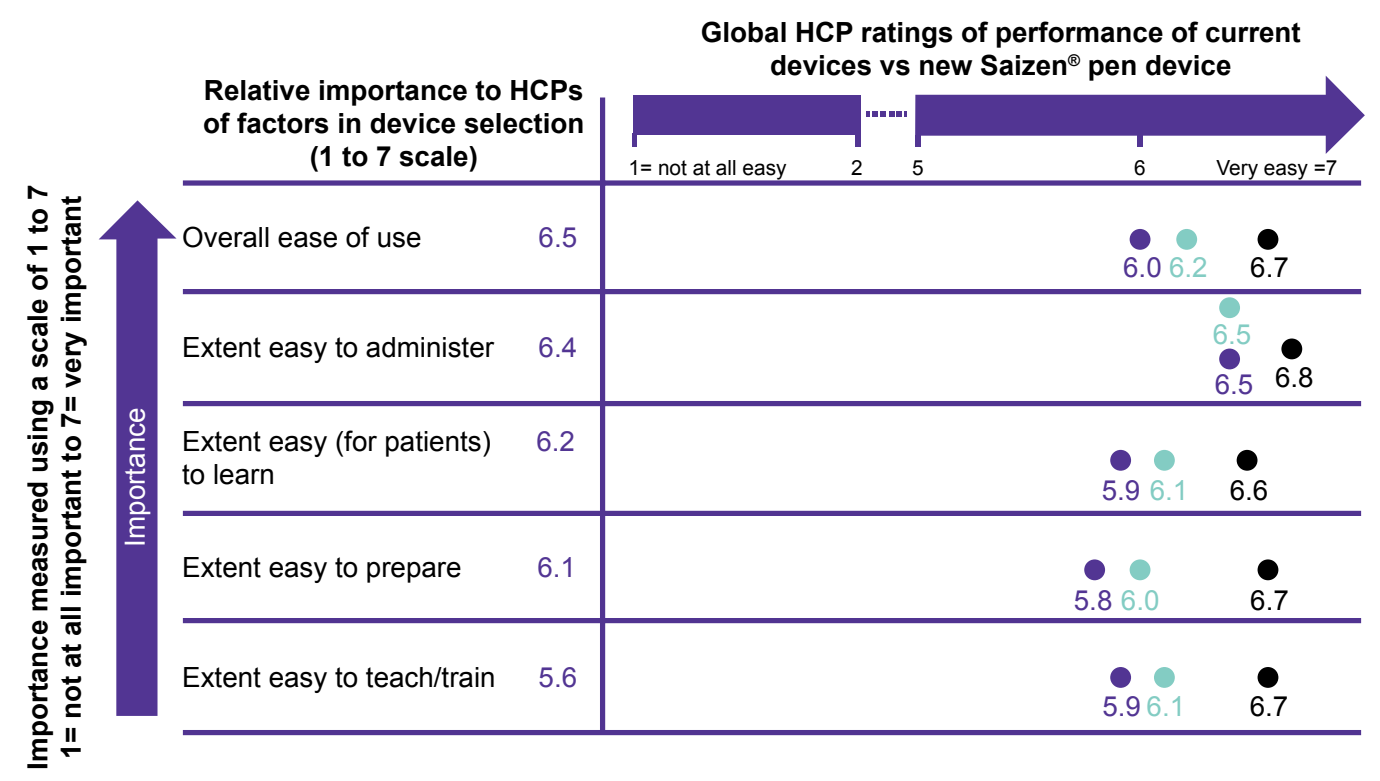

Best rated in market - disposable $(n=33) \bigcirc$ New Saizen ${ }^{\circledR}$ pen $(n=55) \bigcirc$ Best rated in market - reusable $(n=30)$

Figure 4 Global HCP assessment of relative importance of factors in device selection and comparative ratings of device performance.

Notes: Saizen ${ }^{\circledast}$ is manufactured by Merck KGaA, Darmstadt, Germany.

Abbreviation: HCP, health care professional.

home, and they were encouraged to review the materials provided by the manufacturer in advance (DVD, brochures and Quick Reference Guide). Efforts would also be made to ensure the necessary insurance paperwork was in place.

In France and Germany, pen device training sessions commonly last for 20-30 minutes and, as in the USA, might be carried out either by a doctor or a nurse. In Brazil and South Korea, a doctor or nurse provide basic information on administration (eg, angle of injection, pinching the skin appropriately), storage, calculating dose, site of injection and the medication itself at an initial training session lasting 5-20 minutes. This would be followed up by a nurse employed by the distributor or manufacturer who would give more detailed advice on the specific device to be used. These follow-up sessions typically take 20-30 minutes.

The easier a device is to teach or learn how to use, the more effective this brief training is likely to be and the less need there should be for follow-up training. Ease of use is also likely to have a positive impact on long-term adherence to treatment when using a device. This study indicated that the new pen device was easier to use than the current market-leading reusable device and was at least comparable to the market-leading disposable device, which may mean

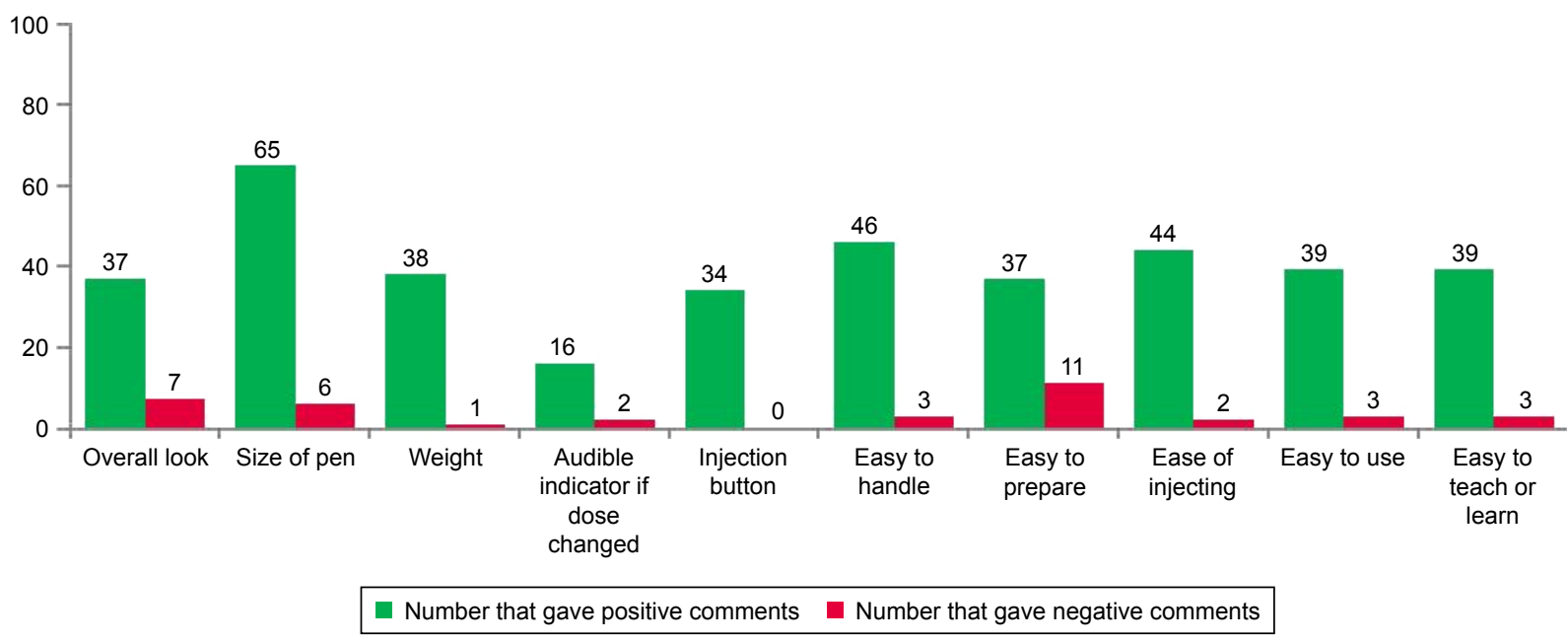

Figure 5 Global HCP Data - number of favorable mentions versus reservations per device attribute for the pen device (selected attributes only). Abbreviation: HCP, health care professional. 
Table 2 Global caregiver/patient rating for performance of the pen device versus their current device - mean scores on a I to 7 scale ( $I=$ not at all easy, $7=$ very easy)

\begin{tabular}{lllll}
\hline Device & $\begin{array}{l}\text { Easy to } \\
\text { learn }\end{array}$ & $\begin{array}{l}\text { Easy to } \\
\text { prepare }\end{array}$ & $\begin{array}{l}\text { Easy to } \\
\text { administer }\end{array}$ & $\begin{array}{l}\text { Overall } \\
\text { ease of use }\end{array}$ \\
\hline $\begin{array}{l}\text { New Saizen }{ }^{\circledR} \text { pen } \\
\text { device }(n=29)\end{array}$ & 5.8 & 6.1 & 6.3 & 6.0 \\
$\begin{array}{l}\text { All current } \\
\text { devices }(n=34)\end{array}$ & 5.8 & 5.2 & 5.5 & 5.8 \\
\hline
\end{tabular}

Notes: Saizen ${ }^{\circledast}$ manufactured by Merck KGaA, Darmstadt, Germany.

that some patients/caregivers will need less overall training in the use of the new device.

In the cohort of patients/caregivers, around half expressed a definite interest (categories 8-10) in asking their HCP about the possibility of switching to the new pen device (and possibly to a new $r$-hGH preparation), and only $16 \%$ were unlikely to make such a request (categories 0-2). Overall, many of the patients/caregivers involved in this study had experience with a different pen device, and many of these would have been using a specific device for a long time and may now be looking to switch. Therefore, the high level of acceptability for the pen expressed by patients and caregivers with experience of injection devices may be a strong indicator of a growing need for this new device with its updated features.

Concerns about the need to calculate any partial doses to make up to the full dose required when the current cartridge did not contain enough $\mathrm{r}$-hGH could be mitigated through effective instruction and reassurance and also by highlighting the cost savings derived from reduced wastage of medication that occurs if partial doses are administered.
The environmental benefits of a reusable device were also viewed favorably by the participants in this study and might offset the advantages of disposable devices reported by HCPs in the ease of preparing, ease of administering and ease of teaching/learning (Table 3; Figure 4). Concerns about the steps of the Quick Reference Guide relating to the plunger mechanism not being clear enough have subsequently been addressed by rewording of this part of the guide, based on the feedback obtained in this study.

This assessment was obtained across the full spectrum of people likely to be involved in r-hGH treatment - nurses, endocrinologists, GHCs, MAs, caregivers and patients from 5 different countries. Trained professionals were recruited to observe and record the comprehensibility of the Quick Reference Guide and the ability of the participants to prepare and use the pen device. However, because of the relatively small sample size of this qualitative study, detailed statistical analyses or conclusions cannot be drawn from the results.

\section{Conclusion}

The prototype pen device successfully met its design objectives and was well accepted by the nurses, endocrinologists, patients and caregivers in this study for its overall ease of use, appearance and functionality. The appearance and ease of use of this new pen device was considered superior to that of the current market-leader reusable pen, and users can be confident of the delivery of the intended dose. Given the importance of adherence to daily r-hGH injections over many years, the perceived acceptability of this pen device could be of benefit to the many patients with GHD-related conditions.

Table 3 Comparison of the new pen device with the leading disposable and reusable injection devices

\begin{tabular}{llll}
\hline Pen type & Saizen ${ }^{\circledR}$ device & Market leader & \multicolumn{1}{c}{$\begin{array}{c}\text { Market leader } \\
\text { disposable }\end{array}$} \\
\hline Feature & Reusable & Reusable & Disposable \\
Easy to prepare & $\checkmark \checkmark$ & $\checkmark$ & $\checkmark \checkmark \checkmark$ \\
Medication is premixed & No & Yes & Yes \\
Dosing window is easy to read & $\checkmark$ & $\checkmark \checkmark \checkmark$ & $\checkmark \checkmark \checkmark$ \\
Ability to dial dose down & $\checkmark$ & $\checkmark$ & $\checkmark$ \\
Manual reset of plunger required & Yes & No & N/A \\
Easy to administer & $\checkmark \checkmark$ & $\checkmark$ & $\checkmark \checkmark \checkmark$ \\
Easy to control injection button & $\checkmark \checkmark \checkmark$ & $\checkmark$ & $\checkmark \checkmark \checkmark$ \\
Option to hide needle & Yes & None & Yes \\
Injection feedback & During (clicking) & & At end (click) \\
& At end (window) & $\checkmark$ & $\checkmark \checkmark \checkmark$ \\
Easy to teach/learn & $\checkmark \checkmark$ & $\checkmark$ & $\checkmark \checkmark \checkmark$ \\
Appealing look and feel & $\checkmark \checkmark \checkmark$ & & \\
\hline
\end{tabular}

Notes: Features of the new Saizen ${ }^{\circledR}$ pen rated as favorable by HCPs and caregivers/patients compared with the reusable and disposable market leaders. The score is derived from the qualitative assessment of the Saizen ${ }^{\circledast}$ pen relative to the current market-leading devices, according to feedback from HCPs, patients and caregivers. Saizen ${ }^{\circledast}$ is manufactured by Merck KGaA, Darmstadt, Germany. Ticks represent the favorability of the features of injection devices (one tick = favorable; two ticks = very favorable; three ticks $=$ highly favorable)

Abbreviations: HCPs, health care professionals; N/A, not applicable. 


\section{A}

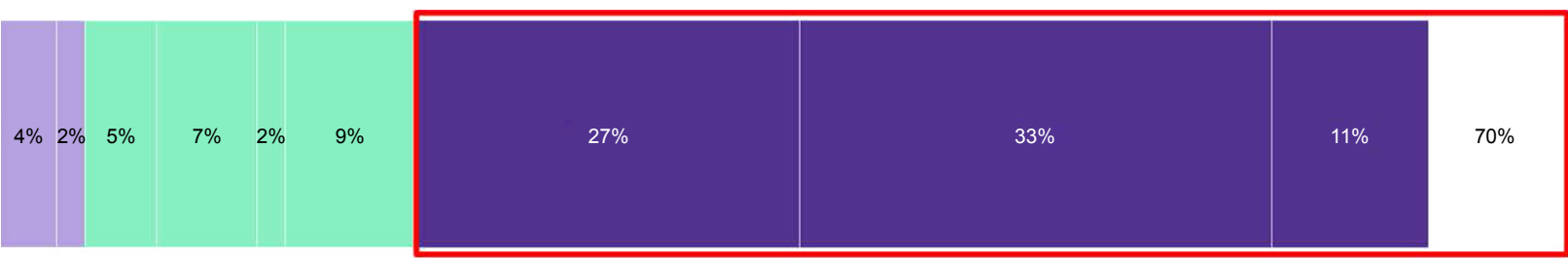

B

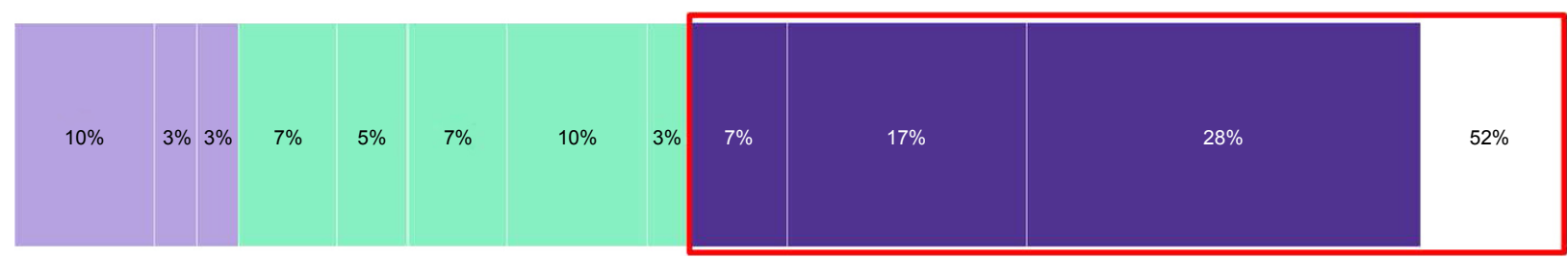

$$
\text { 0= extremely unlikely } \quad \begin{array}{ll|l|l|l|l|l|l|l|l|l|l|}
1 & 2 & 3 & 4 & 5 & 6 & 7 & 8 & 9 & 10=\text { extremely likely }
\end{array}
$$

Figure 6 (A) Likelihood of HCPs to recommend the pen device to patients. (B) Likelihood of caregivers/patients to request the pen device from their HCP. Note: Based on use of a 0 to 10 scale $(0=$ extremely unlikely, $10=$ extremely likely). Abbreviation: HCP, health care professional.

\section{Acknowledgments}

The authors would like to thank the patients, caregivers and HCPs for their participation. The authors would also like to thank Shazada Ali at Double Helix Discovery, London, UK. This study was sponsored by Merck KGaA, Darmstadt, Germany. Saizen ${ }^{\circledR}$ is manufactured by Merck KGaA, Darmstadt, Germany. Medical writing assistance was provided by David Candlish and Steven Goodrick, inScience Communications, UK, and funded by Merck KGaA, Darmstadt, Germany.

\section{Author contributions}

Both authors contributed to the design of the study, data collection and interpretation and the writing and review of the manuscript and agree to be accountable for all aspects of the work.

\section{Disclosure}

MS is an employee of Merck KGaA, Darmstadt, Germany. CA is an independent pharmaceutical market research consultant. The authors report no other conflicts of interest in this work.

\section{References}

1. John M, Koledova E, Kumar KM, Chaudhari H. Challenges in the diagnosis and management of growth hormone deficiency in India. Int J Endocrinol. 2016:2967578.
2. Taddeo D, Egedy M, Frappier JY. Adherence to treatment in adolescents. Paediatr Child Health. 2008;13(1):19-24.

3. De Pedro S, Murillo M, Salinas I, et al. Variability in adherence to rhGH treatment: Socioeconomic causes and effect on children's growth. Growth Horm IGF Res. 2016;26:32-35.

4. Haverkamp F, Johansson L, Dumas H, et al. Observations of nonadherence to recombinant human growth hormone therapy in clinical practice. Clin Ther. 2008;30(2):307-316.

5. Fisher BG, Acerini CL. Understanding the growth hormone therapy adherence paradigm: a systematic review. Hormone Res Paediatr. 2013;79(4):189-196.

6. Rohrer TR, Horikawa R, Kappelgard AM. Growth hormone delivery devices: current features and potential for enhanced treatment adherence. Expert Opin Drug Deliv. 2017;14(11):1253-1264.

7. Kapoor RR, Burke SA, Sparrow SE, et al. Monitoring of concordance in growth hormone therapy. Arch Dis Child. 2008;93(2):147-148.

8. Desrosiers P, O'Brien F, Blethen S. Patient outcomes in the GHMonitor: the effect of delivery device on compliance and growth. Pediatr Endocrinol Rev. 2005;2(Suppl 3):327-331.

9. Raimer-Hall D, Shea HC. Evolution of growth hormone devices. Pediatr Nurs. 2015;41(2):72-77.

10. EphMRA. EphMRA Code of Conduct. European Pharmaceutical Market Research Association; Bromley, Kent, UK 2017.

11. Juster FT. Consumer anticipations and models of durable goods demand. In: Mincer J, ed. Economic Forecasts and Expectations New York: Columbia Universit Press; 1969.

12. Reed ML, Merriam GR, Kargi AY. Adult growth hormone deficiency benefits, side effects, and risks of growth hormone replacement. Front Endocrinol (Lausanne). 2013;4:64

\section{Dovepress}

\section{Publish your work in this journal}

Patient Preference and Adherence is an international, peer-reviewed, open access journal that focuses on the growing importance of patient preference and adherence throughout the therapeutic continuum. Patient satisfaction, acceptability, quality of life, compliance, persistence and their role in developing new therapeutic modalities and compounds to optimize clinical outcomes for existing disease states are major areas of interest for the journal. This journal has been accepted for indexing on PubMed Central. The manuscript management system is completely online and includes a very quick and fair peer-review system, which is all easy to use. Visit http://www. dovepress.com/testimonials.php to read real quotes from published authors. 